\title{
Are There Biologic Factors of Good Prognosis after a Platelet Rich Plasma (PRP) Injection for Tendinopathies?
}

\author{
Libertiaux V ${ }^{1}$, Sancerne $A^{2}$, Le Goff $C^{3}$, Crielaard JM'2,4 and Kaux JF ${ }^{2,4 *}$ \\ ${ }^{1}$ Department of Clinical Sciences, Faculty of Veterinary Medicine, University of Liège, Liège, Belgium \\ ${ }^{2}$ Department of Motricity Sciences, University of Liège, Allée des Sports, B21, 4000 Liège, Belgium \\ ${ }^{3}$ Department of Clinical Chemistry, University and University Hospital of Liège, Belgium. \\ ${ }^{4}$ Department of Physical Medicine and Sports Traumatology, FIFA Medical Centre of Excellence, CHU Liège, Avenue de l'Hôpital, B35, 4000 Liège, Belgium
}

\begin{abstract}
Received: June 1, 2016; Accepted: June 26, 2016; Published: September 21, 2016
*Corresponding author: KAUX Jean-François, Physical Medicine and Sport Traumatology, University Hospital of Liège, Avenue de l'Hôpital, B35, 4000 Liège, Belgium, Tel: +32 436682 41; Fax: +32 436672 30; E-mail: jfkaux@chu.ulg.ac.be
\end{abstract}

\begin{abstract}
Background : The aims of this study were to evaluate the effect of a single PRP injection followed by a standardized reeducation protocol among patients suffering from different tendinopathies, but otherwise healthy, and to determine the biomarkers that constitute good prognosis factors, if any.

Methods : 48 patients suffering from different tendinopathies and refractory to conventional physiotherapy were treated with a single PRP injection. Prior to the injection, a blood sample was drawn and some biological parameters (glycemia, cholesterolemia,...) were measured. A pain assessment was then made using a Visual Analog Scale of pain (VAS) and a pressure algometer. The same assessment was carried out after 6 weeks and 12 weeks when possible.

Results : There is an overall significant improvement in VAS score at the end of the 12 weeks follow- up. However, no correlation was found between the evolution of the clinical scores and the biological parameters measured.

Conclusion: A PRP injection followed by a program of eccentric rehabilitation positively affects the algo-functional scores of patients with tendinopathies who were refractory to conventional physiotherapy, whatever the initial values of the measured biological parameters.
\end{abstract}

Keywords: Tendons ; Platelets Rich Plasma ; Biomarkers

\section{Introduction}

Tendinopathies represent a significant part of musculoskeletal injuries [1]. They are generally caused by microtraumae following a mechanical overload due to work or sport activities. Inflammation, like rhumatoid polyarthritis or spondylarthritis, can also result in tendon injury. Several factors can facilitate the onset of the tendinopathy, which can be either intrinsic or extrinsinc [2]. Amongst the former, we can distinguish between those that can be altered (hyperucemia, hypercholesterolemia, chondrocalcinosis, disthyroidis, degenerative rheumatism, infection,...) [3] and those that cannot (age, gender,...). Examples of extrinsinc factors include unadapted material, bad training planification, or usage of some drugs (quinolones, corticoids,...) [4]. Some patients are refractory to the traditional treatments (including physiotherapy, shockwaves, deep transverse massage, etc.) and thus need another option. Less invasive than surgery, Platelet-Rich Plasma (PRP) injection has become more and more popular over the last decades, particularly in sports medicine [5-7]. In this paper, in addition of presenting the data asserting the effectiveness of injections of reproducible PRP collected from an apheresis machine on different tendinopathies, we introduce another original contribution : the investigation of the possible correlation between several biomarkers and healing evolution to determine the factors of good prognosis, if any.

\section{Material and Methods}

Forty-eight patients (mean age $=49$ y.o +/- 10 y.o, min $=26$ y.o, $\max =77$ y.o. ; 15 women and 33 men) afflicted with different pathologies participated in this study after they gave their informed consent (lateral epicondylitis $(n=20)$, medial epicondylitis $(n=3)$, tendinopathy of the gluteus medius $(n=1)$, patellar tendinopathy $(n=6)$, calcaneal tendinopathy $(n=14)$, and plantar fasciitis $(n=4)$ ). To be recruited, the patients have had to suffer from a chronic tendinopathy for more than 3 months and be refractory to the following classical treatments : non steroidal anti inflammatory drugs, electrotherapy, deep transverse massage, eccentric revalidation and shockwaves therapy. A clinical examination was carried out to confirm the diagnosis and to exclude any other pathology, including metabolic disease. After this first step, a blood sample was drawn and analyzed to quantify the presence of various metabolic biomarkers (glycemia, thyroid hormons, cholesterolemia, uricemia) which are considered to be risk factors of tendinopathies. The initial pain assessment was made using a Visual Analogic Scale (VAS) of pain and a pressure algometer (Commander Algometer, Tech Medical) prior to the PRP collection. The PRP collection and injection protocol used has already been described in previous 
reports [8-10]. Briefly, the platelets are extracted using an apheresis machine (TEC.COM, Fresenius Kabi) which can lead to a reproducible platelet concentration of 850000 units/microlitre, a value approximatively 4 times as high as in the whole blood [11]. 6 millilitres of this PRP were mixed with 300 microlitres of calcium chloride to activate the platelets. The injection was performed under ultrasound guidance and without local anaesthesia as it could decrease PRP healing effectiveness. The physiotherapy started 5 days after the injection, 3 times a week for 6 weeks [12]. The pain was reassessed 6 weeks and 12 weeks after the injection. For the statistical processing of these results, a Shapiro-Wilk test was made to evaluate the normal distribution of the data and a paired Welch test was used to compare the results of the VAS and the algometer at the different time points. Linear mixed effects models (nlme package, RStudio, Rstudio Inc.) were used to investigate the potential relationship between the biological parameters and the clinical evolution (evaluated by VAS and algometer scores).

\section{Results}

When combining the results for all the tendons, a significant decrease of the VAS score was noted after 6 weeks (VAS0 = 4.81, $\triangle$ VAS0 $-6=-0.84 ; \mathrm{p}=0.007$ ) and after 12 weeks (VAS0 $=$ $4.81, \triangle$ VAS0-12 $=-1.1 ; \mathrm{p}=0.01$ ). The result of the algometer also improved significantly between the 6 th and the 12th week (Algo6 $=52.8 \mathrm{~N} / \mathrm{cm}^{2}, \Delta$ Algo6-12 = $12.9 \mathrm{~N} / \mathrm{cm}^{2} ; \mathrm{p}<0.05$ ). Another way to look at the results is to observe that $70 \%$ of the patients showed a positive effect following the PRP injection with a decrease of pain of at least 1 point on the VAS (Fig. 1). For $25 \%$ of them, the decrease was at least 4 points. Independently, $40 \%$ of the patients saw their algometer result increase by more than 30N/ $\mathrm{cm}^{2}$ by the end of the 12 week follow-up (Fig. 2). A tendon-specific analysis was also performed. Statistically significant results were only found for the groups of patients suffering from epycondilitis $(\triangle \mathrm{VAS} 0-12=-1.69, \mathrm{p}<0.005)$ and Achilles tendinopathy ( $\triangle \mathrm{VAS} 0$ $6=-1.96, \mathrm{p}<0.005$ ). The sample size of the other tendinopathies was too small to observe a statistically significant evolution.

A possible correlation between hypercholesterolemia and hyperglycemia and the evolution of VAS and algometer results was investigated (all the other biomarkers were in the normal range for all the patients). Such a correlation was not observed. Furthermore, no statistically significant differences in $\triangle$ VAS0-12 and $\Delta$ Algo 0-12 were found after clustering the patients into two groups, one with normal and one with elevated cholesterolemia (Fig. 3) and glycemia (Fig. 4) .

\section{Discussion}

This study quantitatively confirmed the effectiveness of a single PRP injection followed by an eccentric revalidation for patients suffering from various tendinopathies refractory to conventional treatments, as shown by the $23 \%$ decrease in $\triangle$ VAS0-12 and $24 \%$ increase in $\Delta$ Algo6-12. The tendon-specific results have too few data points to show statistical significance, but the trend observed is encouraging and confirms the results found in the literature. Indeed, in the case of epicondylitis, an algofunctional improvement was noticed as previously reported.

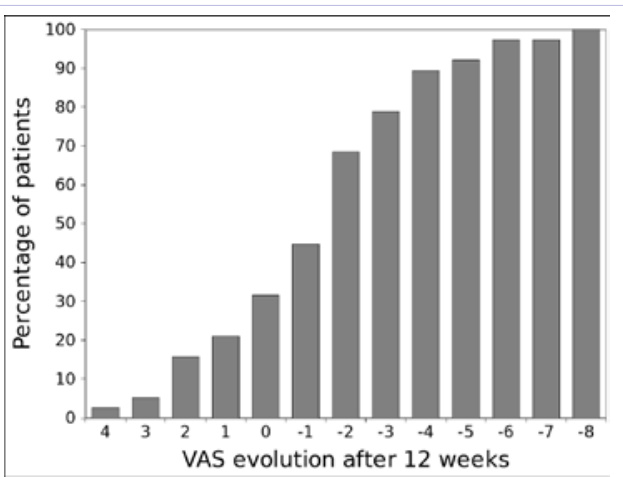

Figure 1: Cumulative histogram of the evolution of the VAS score for the 48 patients over the 12 weeks clinical trial. The pain decreased for $70 \%$ of the patients, $10 \%$ saw no difference and the pain increased for $20 \%$.

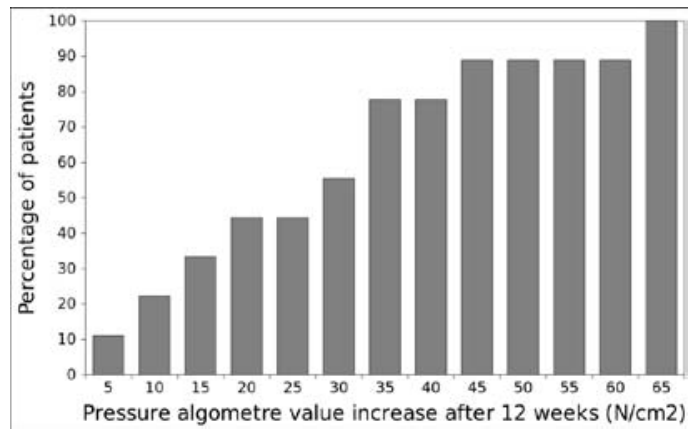

Figure 2: Cumulative histogram of the evolution of the algometre score for the 48 patients over the 12 weeks clinical trial. All patients saw an improvement of their score which reached $30 \mathrm{~N} / \mathrm{cm}^{2}$ for more than $50 \%$.

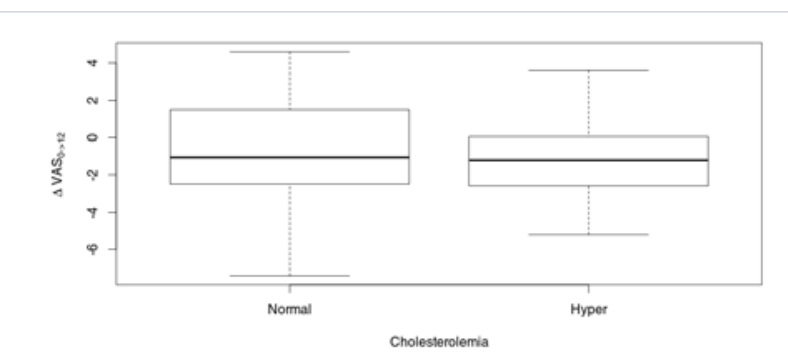

Figure 3: Evolution of the VAS score over the 12 weeks clinical trial with respect to cholesterolemia - whisker plot. 23 patients had a normal level and 24 presented hypercholesterolemia.

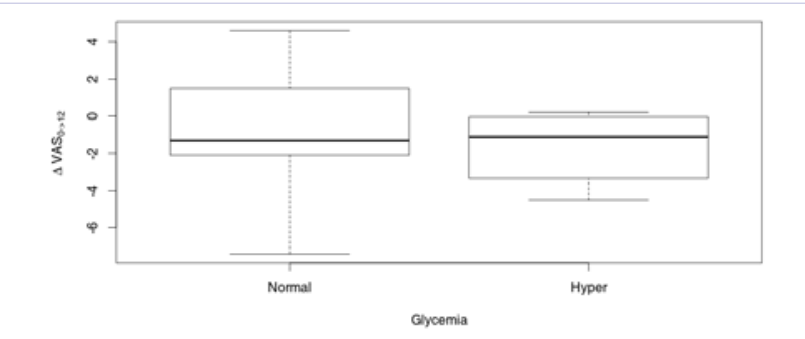

Figure 4: Evolution of the VAS score over the 12 weeks clinical trial with respect to glycemia - whisker plot. 28 patients had a normal level and 18 presented hyperglycemia. 
$[13,14]$. The follow-up in those studies lasted 24 weeks and 28 months respectively. As for Achilles tendinopathy, several other studies also demonstrated a decrease in pain following a PRP injection [15-17]. To the authors' opinion, the lack of significant results in the tendon-specific part of the present study is therefore due to the small number of patients recruited. Furthermore, no follow-up information could be obtained from several patients. Despite all the studies mentioned point in the same direction, it is difficult to compare them as the PRP collection protocols might be quite different. Indeed, the collection itself might be performed using laboratory or commercial machines, or also an apheresis machine. The amount of platelets will then vary according to the extraction method and is not repeatable in the case of the commercial technique $[18,19]$. This study presents, to the best of the authors' knowledge, the first attempt to correlate biomarkers as factors of good prognosis to the evolution of the recovery. Hyperglycemia and especially type II diabetes, leads to a disorganization of collagen fibers, and an increase in tendons thickness and volume [20] ; hyperthyroidism increases collagen catabolism and hypothyroidism leads to a decreased collagen metabolism [21]; hypercholesterolemia impairs the tendon extra cell matrix and thus alters its biomechanical properties [22] and finally, hyperucemia could cause an inflammation provoking the degeneration of the tendon tissue [23]. It might then be surprising that no correlation was found between the values of the biological markers and the evolution of the tendons healing evaluated by the VAS and the algometer. However, one has to distinguish between the correlation between the values of the markers and the susceptibility of developing a tendinopathy compared to the susceptibility of slower healing once the pathology has triggered. The former is out of the scope of the present report. From our results, it seems that the initial glycemia and cholesterolemia don't have any influence on the prognosis of the evolution of the tendinopathy. In a previous report [8], a young age of the patients ( $<30$ years old) and a better strength of the quadriceps before infiltration were identified as good prognosis factors to recover from a jumper's knee while diabetes was a bad one [24].

Further studies should focus on comparing the effectiveness of the PRP treatment for different tendinopathies, which couldn't be established in the present study because of the relative small number of patients afflicted with different pathologies. The total number of patients was however sufficient to obtain statistically significant results regarding the effectiveness of a single PRP injection in the treatment of tendinopathies. Other good prognosis factors, like biomechanical ones, should be investigated too.

\section{Acknowledgements}

This study was supported by the Lejeune-Lechien Founds of the Léon Frédéricq Foundation.

\section{References}

1. Forde MS, Punnett L, Wegman DH. Prevalence of musculoskeletal disorders in union ironworkers. J Occup Environ Hyg. 2005;2(4):203212. DOI:10.1080/15459620590929635.

2. Kaux JF, Forthomme B, Goff CL, Crielaard JM, Croisier JL. Current opinions on tendinopathy. J Sports Sci Med. 2011;10(2):238-253.

3. Ackermann PW, Renström P. Tendinopathy in sport. Sports Health. 2012;4(3):193-201. DOI: 10.1177/1941738112440957.
4. Fournier PE, Rappoport G. [Tendinopathy: physiopathology and conservative treatment]. Rev Med Suisse. 2005;1(28):1840-1842, 1845-1846.

5. Smets F, Croisier JL, Forthomme B, Crielaard JM, Kaux JF. Clinical applications of platelet-rich plasma (PRP) in tendon lesions: A literature review Science \& Sport. 2012;27(2):141-153.

6. Kaux JF, Drion P, Croisier JL, Crielaard JM. Tendinopathies and plateletrich plasma (PRP): from pre-clinical experiments to therapeutic use. J Stem Cells Regen Med. 2015;11(1):7-17.

7. Sampson S, Gerhardt M, Mandelbaum B. Platelet rich plasma injection grafts for musculoskeletal injuries: a review. Curr Rev Musculoskelet Med. 2008;1(3-4):165-174. doi:10.1007/s12178-008-9032-5.

8. Kaux JF, Croisier JL, Bruyere O, Rodriguez De La Cruz C, Forthomme B, Brabant G, et al. One injection of platelet-rich plasma associated to a submaximal eccentric protocol to treat chronic jumper's knee.J Sports Med Phys Fitness. 2015;55(9):953-961.

9. Kaux J-F, Bruyère O, Croisier J-L, Forthomme B, Le Goff C, Crielaard J-M. One-year follow-up of platelet-rich plasma infiltration to treat chronic upper patellar tendinopathies. Acta Orthop Belg. 2015 Jun;81(2):251-6.

10. Kaux JF, Croisier JL, Forthomme B, Le Goff C, Buhler F, Savanier B, et al. Using platelet-rich plasma to treat jumper's knees: Exploring the effect of a second closely-timed infiltration. J Sci Med Sport. 2016;19(3):200204. doi: 10.1016/j.jsams.2015.03.006.

11. Moog R, Franck V, Pierce JA, Müller N. Evaluation of a concurrent multicomponent collection system for the collection and storage of WBC-reduced RBC apheresis concentrates. Transfusion. 2001;41(9):1159-1164

12. Kaux JF, Forthomme B, Namurois MH, Bauvir P, Defawe N, Delvaux F, et al. Description of a standardized rehabilitation program based on sub-maximal eccentric following a platelet-rich plasma infiltration for jumper's knee. Muscles Ligaments Tendons J. 2014;4(1):85-89.

13. Mishra AK, Skrepnik NV, Edwards SG, Jones GL, Sampson S, Vermillion DA, et al. Efficacy of platelet-rich plasma for chronic tennis elbow: a double-blind, prospective, multicenter, randomized controlled trial of 230 patients. Am J Sports Med. 2014;42(2):463-471. doi: $10.1177 / 0363546513494359$.

14. Hechtman KS, Uribe JW, Botto-vanDemden A, Kiebzak GM. Plateletrich plasma injection reduces pain in patients with recalcitrant epicondylitis. Orthopedics. 2011;34(2):92. doi: 10.3928/0147744720101221-05

15. Murawski CD, Smyth NA, Newman H, Kennedy JG. A single plateletrich plasma injection for chronic midsubstance achilles tendinopathy: a retrospective preliminary analysis. Foot Ankle Spec. 2014;7(5):372376. doi: 10.1177/1938640014532129.

16. Owens RF, Ginnetti J, Conti SF, Latona C. Clinical and magnetic resonance imaging outcomes following platelet rich plasma injection for chronic midsubstance Achilles tendinopathy. Foot Ankle Int. 2011;32(11):1032-1039.

17. Mautner K, Colberg RE, Malanga G, Borg-Stein JP, Harmon KG, Dharamsi AS, et al. Outcomes after ultrasound-guided platelet-rich plasma injections for chronic tendinopathy: a multicenter, retrospective review. PM R. 2013;5(3):169-175. doi: 10.1016/j.pmrj.2012.12.010.

18. Kaux JF, Le Goff C, Seidel L, Péters P, Gothot A, Albert A, et al. [Comparative study of five techniques of preparation of platelet-rich plasma]. Pathol Biol (Paris). 2011;59(3):157-160. doi: 10.1016/j. patbio.2009.04.007. 
19. Kaux JF, Le Goff C, Renouf J, Peters P, Lutteri L, Gothot A, et al. Comparison of the platelet concentrations obtained in platelet-rich plasma (PRP) between the GPS ${ }^{\mathrm{TM}}$ II and GPS ${ }^{\mathrm{TM}}$ III systems. Pathol Biol (Paris). 2011;59(5):275-277. doi: 10.1016/j.patbio.2010.11.002.

20. de Oliveira RR, Lemos A, de Castro Silveira PV, da Silva RJ, de Moraes SR. Alterations of tendons in patients with diabetes mellitus: a systematic review. Diabet Med. 2011;28(8):886-895. doi: 10.1111/j.14645491.2010.03197.x.

21. Oliva F, Berardi AC, Misiti S, Maffulli N. Thyroid hormones and tendon: current views and future perspectives. Concise review. Muscles Ligaments Tendons J. 2013;3(3):201-203.
22. Abboud JA, Beason DP, Soslowsky LJ. Emerging ideas: the effect of hypercholesterolemia on tendons. Clin Orthop Relat Res. 2012;470(1):317-320. doi: 10.1007/s11999-010-1709-6.

23. Abate M, Schiavone C, Salini V, Andia I. Occurrence of tendon pathologies in metabolic disorders. Rheumatology (Oxford). 2013;52(4):599-608. doi:10.1093/rheumatology/kes395.

24. Kaux JF, Croisier JL, Léonard P, Le Goff C, Crielaard JM. Exuberant inflammatory reaction as a side effect of platelet-rich plasma injection in treating one case of tendinopathy. Clin J Sport Med. 2014;24(2):150152 . 